\title{
Treatment of pain post-brachial plexus injury using high-frequency spinal cord stimulation
}

This article was published in the following Dove Press journal: Journal of Pain Research

\section{Daniela Floridia \\ Francesco Cerra \\ Giuseppe Guzzo \\ Silvia Marino \\ Nunzio Muscarà \\ Francesco Corallo \\ Alessia Bramanti \\ Antonino Chillura \\ Antonino Naro}

IRCCS Centro Neurolesi Messina, Messina, Italy
Correspondence: Daniela Floridia IRCCS Centro Neurolesi Messina, Via Salita Contino Messina due, lotto L pal. 32, Messina, Italy

Tel +393358392578

Fax +39 902937201

Email danielafloridia@yahoo.it
Purpose: Brachial plexopathy can sometimes cause severe chronic pain. There are many possible treatments for such neuropathic pain, including neuromodulation. However, rigorous scientific evidence on the usefulness of spinal cord stimulation (SCS) is still scarce. Here, we report the use of high-frequency $(10 \mathrm{kHz}$ ) SCS (HFSCS) in a patient with brachial plexus injury (root avulsion).

Objective: To assess the efficacy of HFSCS in root avulsion and to investigate the putative neurophysiological mechanisms of HFSCS.

Methods: A 32-year-old woman visited our center following an iatrogenic brachial plexus injury. She underwent traditional, paresthesia-inducing, tonic SCS with cervical lead placement. She reported that stimulation-induced paresthesia was uncomfortable, without any pain reduction. After the successful trial of HFSCS, the patient was assessed at 1 month (T1) and 6 months (T6) after HFSCS implantation with pain and quality of life (QoL) scales. Moreover, she underwent a neurophysiological assessment (somatosensory evoked potentials [SEPs], reciprocal inhibition [RI], pain-motor integration [PMI], and the habituation of intraepidermal electrical stimulation-induced evoked potentials [IEPs]) with the stimulator switched on and switched off at T6.

Results: The patient reported $100 \%$ paresthesia-free pain relief, a consistent improvement of QoL, and a complete discontinuation of her previous pain treatment at T1 and T6. Moreover, we found suppression of SEPs, restored habituation of IEPs, and strengthening of RI and PMI. Conclusion: This is the first report to illustrate the usefulness and safety of HFSCS for treating root avulsion in a patient with failed tonic SCS. Our data indicate that HFSCS may either block large-diameter fibers or stimulate medium-/small-diameter fibers, thus inducing analgesia without paresthesia, probably by reducing the activation of the wide-dynamic-range neurons. Moreover, HFSCS seems to modulate spinal inhibitory mechanisms and the descending corticospinal inhibitory output. Thus, HFSCS can be an effective option for treating refractory pain following root avulsion.

Keywords: Mechanism, root, avulsion, chronic, pain, neuromodulation

\section{Introduction}

Spinal cord stimulation (SCS) is a viable alternative for controlling neuropathic pain when pharmacological and surgical treatments are not effective, particularly for chronic, intractable back pain or back pain resulting from failed back surgery syndrome, complex regional pain syndrome, secondary neuropathic pain due to peripheral nerve injury, and posttraumatic brachial plexopathy. ${ }^{1,2}$ Spinal cord neuromodulation systems are composed of a lead, located in the epidural space using a Tuohy needle, 
connected to a subcutaneously implantable pulse generator (IPG), which contains power source and electronic unit to deliver programmable stimulation. Traditional SCS produces paresthesia, experienced by the patient as a tingling sensation, overlapping the target area, namely, the area of pain. Recently, paresthesia-free high-frequency SCS (HFSCS), usually from 1 to $10 \mathrm{kHz}$, has been introduced for treating chronic pain and has shown good efficacy. ${ }^{3}$

The root avulsion of the brachial plexus is usually caused by traction or a penetrating injury of the branches forming the brachial plexus. C5 and C6 are the most affected roots, causing a complete or limited loss of arm, forearm, and hand motility, depending on the amount of fibers compromised. The involvement of C7 and C8 inevitably generates a paralysis of the hand. Brachial plexopathy may occur from different causes, including upper limb/shoulder traction, penetrating wound, neoplastic infiltration, and procedural iatrogenic complications. Notably, brachial plexopathy can sometimes cause severe chronic pain and disability.

Neuropathic pain associated with brachial plexopathy is one of the most difficult complications to treat, even resulting in the patient's request to amputate the limb. Even though there are many possible therapeutic options for neuropathic pain due to brachial plexopathy, including medication, physical therapy, nerve blocks, and SCS, these options are not always successful. Here, we report the use of HFSCS at $10 \mathrm{kHz}$ in a patient who sustained a brachial plexus injury (root avulsion).

\section{Case report}

The patient was a 32-year-old female who sustained an iatrogenic brachial plexus trunk injury as a consequence of the blockage of the plexus during anesthesia for a surgery of the right shoulder capsule in November 2015. She experienced pain in the right shoulder and upper arm and limited motility of the right hand. She was treated with anti-inflammatory agents, corticosteroids, and anticonvulsants, but without benefits. In January 2016, the patient received, at another hospital, a cervical spinal cord neurostimulator implant (lowfrequency SCS) with one lead positioned at the C4-C5 level. Given that low-frequency SCS was not much beneficial, she started to take painkillers up to November 2016, when she visited our center due to continual pain in the right shoulder and upper arm, combined with a numb sensation in the acromial region, superficial allodynia in the subclavicular region, and deep allodynia in the supraclavicular region (Figure 1). The numeric rating scale (NRS) $(0$, no pain; 10 , worst pain imaginable) score was 8 . The Barthel Index score at baseline was $100 / 100$. The radiographic examination of the cervical spine showed electrode positioning at the C4-C5 level (Figure 2A). The electromyogram (EMG) showed normal sensory amplitudes in the examined nerves and a severe neurogenic damage of the muscles innervated by $\mathrm{C} 5-\mathrm{C} 6$, thus suggesting a preganglionic dysfunction according to Millesi classification. The somatosensory evoked potentials (SEPs) generated by stimulating the median nerve from the middle finger were normal, whereas those obtained from the thumb and index were absent (both cervical and cortical components). Ultrasound images of the brachial plexus showed C5 and C6 nerve roots as curvilinear hypoechoic structures exiting from the neural foramina, whereas the primary upper right trunk at the supraclavicular region showed an inhomogeneous ecostructure with diffuse hyperechogenic trauma with internal blurred hypoechogenic areas and slightly increased dimensions compared to that of the contralateral side.

Given that tonic SCS was ineffective, the patient underwent an HFSCS trial period with the lead positioned at the C2-C3 level (Figure 2B). We opted to stimulate the spinal cord at the $\mathrm{C} 2-\mathrm{C} 3$ level because this site of stimulation may affect the $\mathrm{C} 4$ level where the anastomotic descendant branch connects with the upper primary trunk of the brachial plexus originating from the union of the front branch of $\mathrm{C} 5$ and C6 that was the cause of pain and allodynia. In December 2016 , the patient reported $100 \%$ pain relief $(\mathrm{NRS}=0)$, did

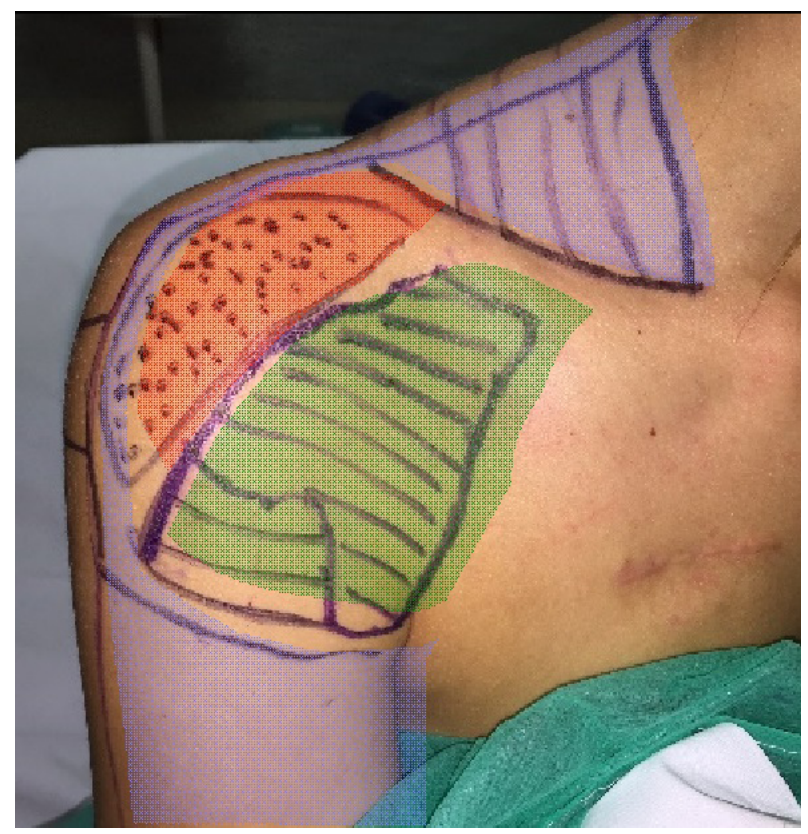

Figure I Patient with iatrogenic brachial plexus trunk injury as a consequence of the blockage of the plexus during anesthesia for a surgery of the right shoulder capsule.

Note: Green area: superficial allodynia area; purple area: deep allodynia area; red area: numbness. 
A

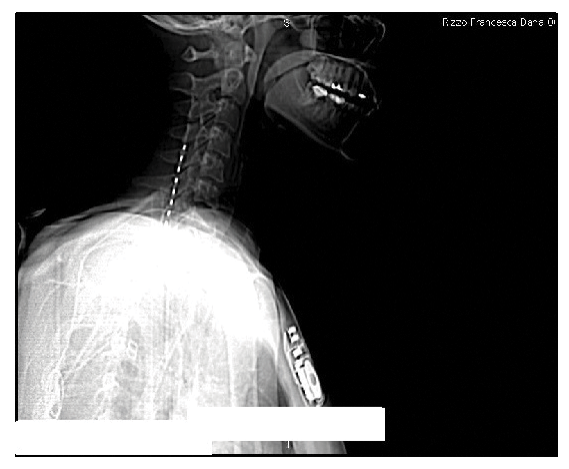

B

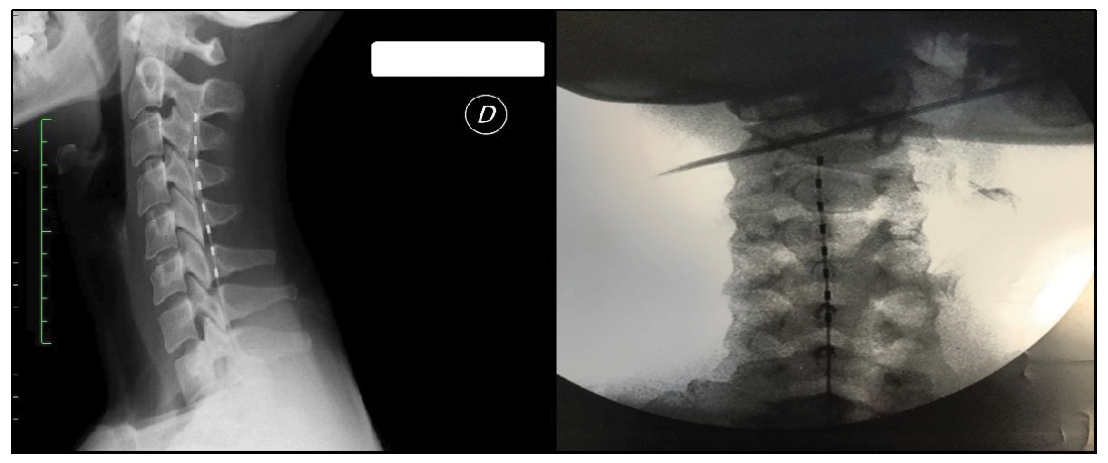

Figure 2 (A) Cervical (C5-C6) lead position with tonic SCS. (B) Cervical (C2-C3) lead position with HFSCS.

Abbreviations: SCS, spinal cord stimulation; HFSCS, high-frequency SCS.

Table I QoL scores at baseline (T0) and after 6 months from HFSCS (T6)

\begin{tabular}{|l|l|l|}
\hline QoL & T0 & T6 \\
\hline Physical activity & 50 & 80 \\
Physical limitations & 60 & 100 \\
Physical pain & 72 & 90 \\
General health & 45 & 80 \\
Vitality & 50 & 70 \\
Social activities & 75 & 75 \\
Emotional limitations & 100 & 100 \\
Mental health & 100 & 100 \\
\hline
\end{tabular}

Abbreviation: QoL, Quality of life; HFSCS, high-frequency SCS.

not complain of superficial and deep allodynia, and showed no change in the numbness and partial motor deficiency. This improvement was maintained after the permanent implant of the IPG (February 2017), resulting in withdrawal of painkillers at 1-month follow-up (March 2017). According to the Minnesota Multiphase Personality Inventory, the patient had a "normal" profile, with an improved quality of life (QoL) rate compared to that at 6 months before (Table 1).

To study the putative neurophysiological basis supporting the aftereffects of HFSCS, we tested the following parameters in August 2017 (T6) with SCS on and off in a single session: 1) SEPs depending on $A \beta$ and $A \delta$ afferents, whose amplitude modulation is a marker of SCS efficiency, ${ }^{4}$ 2) after 1 hour, we measured reciprocal inhibition (RI), which represents the presynaptic inhibition of the Ia afferent fibers, thus indirectly quantifying the glycinergic tone at the spinal level, ${ }^{5}$ and 3 ) after 1 hour, we estimated pain-motor integration (PMI) at the cortical level, which represents the sensory-motor integration processes related to the effects of nociceptive inputs onto motor output generation in primary sensory and motor areas. ${ }^{6}$
To test $\mathrm{A} \beta$ afferents, we recorded standard SEPs. We recorded the cortical SEPs in response to right median nerve stimulation at the wrist by using scalp electrodes placed at $\mathrm{CP} 3$ referred to $\mathrm{CP} 4$. SEPs were evoked by a bipolar transcutaneous electrical stimulation applied to the median nerve, with the cathode placed proximal and the anode 3 $\mathrm{cm}$ distal. We delivered biphasic, square-wave pulses with $0.1 \mathrm{~ms}$ pulse width at $3 \mathrm{~Hz}$, with a stimulus intensity able to produce a stable muscle twitch. Two runs of 500 stimuli on average were recorded. SEPs were recorded with the stimulator switched on and immediately after switching off the stimulator. To test $\mathrm{A} \delta$ afferents, we used an intraepidermal electrical stimulation (IES) approach. IES-induced evoked potentials (IEPs) were recorded using a surface disk electrode placed at $\mathrm{Cz}$ referred to $\mathrm{Fz}$, according to an electrode montage that usually allows for recording a clear N2-P2 complex. In analogy to the technique of Inui et $\mathrm{al}^{7}$ and Mouraux et al, ${ }^{8}$ we inserted a pair of thin monopolar needles, $1 \mathrm{~mm}$ spaced, into the epidermis of the C5-C6 territory in the forearm, through which two constant-current square-wave pulses $(0.5$ $\mathrm{ms}$ pulse width) were delivered at an interstimulus interval of 10 ms (DS5; Digitimer, Welwyn Garden City, UK). The stimulation intensity was set to twice the perceptual threshold. The paired stimuli were delivered every 5 seconds, in order to induce habituation phenomenon, that is, a progressive decrease in the evoked response amplitude during the repetitive application of nociceptive stimuli, as demonstrated by using laser-evoked potentials. ${ }^{9}$

RI was studied using a conditioning test paradigm. ${ }^{10}$ The test stimulus (eliciting H-reflex) was delivered using surface disk electrodes wired to an electric stimulator (DS5; Digitimer). The cathode was placed on the median nerve at the elbow (constant-current square-wave pulses, $1 \mathrm{~ms}$ pulse 
width), and the anode was placed on the biceps brachii tendon. EMG activity was recorded from the flexor radialis carpi (FRC) muscle. The test H-reflex amplitude was maintained at $15 \%-20 \%$ of the amplitude of the maximum motor response for the target muscle. ${ }^{11}$ Conditioning stimuli were delivered before the test stimulus of $20 \mathrm{~ms}$ by using a similar stimulation setup, but placing the electrode pairs $2 \mathrm{~cm}$ distal at the cubital tunnel (median nerve) with respect to the test electrode pair. The conditioning stimulus amplitude was set to motor threshold of the flexor carpi ulnaris muscle. Ten conditioned and ten unconditioned stimuli (test only) were recorded and averaged offline. RI was expressed as percentage of unconditioned H-reflex amplitude.

PMI was measured using a transcranial magnetic stimulation (TMS) paradigm paired to IES. First, motor evoked potentials (MEPs) were recorded by delivering magnetic monophasic stimuli through a standard figure-of-eight coil (external wing $9 \mathrm{~cm}$ in diameter) wired to a high-power Magstim 200 Stimulator (Magstim, Whitland, UK). ${ }^{12}$ The coil was placed over the optimum scalp position (hot spot) to elicit wider MEPs from the FRC of the right upper limb. As the handle of the coil was pointing back and deviated away from the midline of $45^{\circ}$, we induced posteroanterior currents in the brain. We preliminarily evaluated the resting motor threshold (RMT), defined as the smallest stimulus intensity that can evoke a peak-to-peak MEP of $50 \mu \mathrm{V}$ in the target muscle at rest, in at least five of ten consecutive tracks. Electromyographic activity was recorded through $\mathrm{Ag}-\mathrm{AgCl}$ surface electrodes applied to the target muscle by using a classic belly-tendon montage. Signals were amplified and filtered (from $32 \mathrm{~Hz}$ to $1 \mathrm{kHz}$ ) using a Digitimer D150 Amplifier, and stored using a sampling frequency of $10 \mathrm{kHz}$ on a personal computer for offline analysis (Signal Software; Cambridge Electronic Design, Cambridge, UK).

We then employed a paired-pulse protocol to assess PMI. A conditioning stimulus, one that was used to evoke IEPs, preceded the test stimulus, one that was used for evoking an MEP, of an individually adapted interval that yielded the maximal MEP suppression. The relative change in MEP amplitude induced by the SCS was taken as a measure of PMI strength. The mean amplitude of ten conditioned MEPs was expressed as percentage of the mean amplitude of the unconditioned one.

\section{Results}

The patient reported immediate benefit during the trial phase, with an NRS of 0 and the disappearance of superficial and deep allodynia, leaving both the numb area and the partial motor deficit unchanged. After 1 week of permanent implant, the drug therapy was withdrawn. At 6 months postimplant, the improvement in pain relief was maintained, and QoL and duration of sleep were also improved.

A $\beta$-SEPs were reduced in amplitude after HFSCS was switched on. Yet, SEPs showed an immediate recovery when the stimulator was switched off. Conversely, IEPs did not significantly change in latency and amplitude with the stimulator on and off $( \pm 10 \%)$. However, a habituation to stimuli of approximately $-25 \%$ was present only when HFSCS was switched on. Moreover, the stimulation-on condition strengthened RI to approximately $40 \%$ as compared to the stimulation-off condition. Similarly, PMI increased by approximately $20 \%$ (ie, potentiated, as indicated by the MEP amplitude suppression) in the stimulation-on vs stimulationoff condition.

\section{Discussion}

HFSCS is proposed as an alternative treatment for chronic pain syndromes that are nonresponsive to traditional SCS. In fact, HFSCS has demonstrated significant clinical evidence with superior results in pain relief as compared to conventional SCS, with $80 \%$ pain relief outcomes..$^{13}$ Our case report suggests the safety and efficacy of cervical HFSCS in C5-C6 root avulsion. The former SCS device with the positioning of the lead at the midline at $\mathrm{C} 4-\mathrm{C} 5$ resulted in inefficient pain relief (20\%). Moreover, SCS-induced paresthesia was poorly tolerated. Conversely, HFSCS with the positioning of the electrode at $\mathrm{C} 2$ provided the patient with immediate benefit and a clear regression of the symptoms. In addition, this approach relieved the patient from extremely uncomfortable paresthesia perceived in correlation with head movements. However, HFSCS did not address the numbness reported by the patient.

Despite its efficacy, the neurophysiological underpinning of HFSCS is still poorly understood. It has been postulated that the traditional, low-frequency SCS exerts pain relief through retrograde stimulation of axons in the dorsal column of the spinal cord and anterograde stimulation of the descending inhibition of wide-dynamic-range (WDR) neurons in the lamina $\mathrm{V}$, which are responsible for neuropathic pain. ${ }^{14-16}$ The traditional, low-frequency SCS-induced paresthesia, an uncomfortable vibration-type sensation, however, matches treatment with pain location. Paresthesia is thought to depend on the abovementioned anterograde axonal stimulation of the outer large-diameter fibers of the dorsal columns, which carry such a vibration-type sensation. ${ }^{17}$

Conversely, HFSCS achieves analgesia without paresthesia; this may be because of a local block of the low-threshold 
large fibers within the lemniscal pathway rather than generating traveling impulses. ${ }^{18,19}$ Our data are consistent with this hypothesis, as indicated by the reduction in SEP amplitude. Moreover, it is likely that our stimulation paradigm did not generate an action potential traveling orthodromically and antidromically along the lemniscal pathway (as instead occurs in the traditional SCS), given that the intensity of HFSCS was set below the threshold for evoking paresthesia. Thus, the absence of the action potential suggests that the SEP inhibition mechanism is located very close to the point where the electrode is located (ie, in the stimulation area) and thus has a segmental action. Specifically, the stimulation of the spinal cord at the $\mathrm{C} 2-\mathrm{C} 3$ level performed in this study may affect the $\mathrm{C} 4$ level, where the anastomotic descendant branch connects with the upper primary trunk of the brachial plexus originating from the union of the front branch of $\mathrm{C} 5$ and $\mathrm{C} 6$.

SEP amplitude reduction is a common finding in different SCSs. Previous studies investigating tonic SCS have indicated different neural interactions (eg, postsynaptic modulation) and/or the release of specific key neurotransmitters and neuropeptides that may relieve pain. ${ }^{13}$ In keeping with the gross clinical similarity of application, HFSCS may act on similar neural structures but may differently modulate them. In fact, given that only large fibers in the dorsal columns can be activated by tonic SCS and most of the fibers in the superficial dorsal columns are smaller than $7 \mu \mathrm{m}$, it is unlikely that HFSCS may reach the portions of the spine where large fibers are located. In this context, it has been recently shown that HFSCS reduces more A $\delta$ fiber-related evoked potentials than tonic SCS. ${ }^{20}$ In keeping with this issue, we may hypothesize that HFSCS could modulate the elaboration of A $\delta$ fiber inputs likely at the spinal level. In fact, we did not observe any significant change in IEP amplitude, while we found a restored habituation phenomenon. These findings suggest that HFSCS may entrain, rather than block, medium- and small-diameter fibers within the dorsal column (as the large fibers have a lower blocking threshold than smaller fibers and are therefore preferentially blocked), where paresthesia is not evoked (lamina II and III). ${ }^{21}$ Such entrainment could reduce, in turn, lamina V WDR cell signaling, probably by means of a complex interplay between nociceptive-specific projection neurons and primary afferent-derived excitatory/ inhibitory drives to the spinal cord dorsal horn, thus influencing the transmission of nociceptive inputs. ${ }^{16,22}$ Therefore, even if the $A \beta$ fibers, once inhibited, through gate dynamics and stimulation effects on the axon, do not affect the inhibitory interneurons and do not produce paresthesia, thus allowing the pain information to travel along the medulla, the modulation of the $\mathrm{A} \delta$ fiber transmission (that does not produce paresthesia) may be the reason why there is no transmission of painful impulse. The complex effects of SCS at the segmental level is also suggested by the local increase in the activity of inhibitory interneurons, as suggested by the potentiation of RI, which is an index of glycinergic function at the spinal level (which receives input from low-threshold $A \beta$ fibers as well). As this is the first time that spinal glycinergic tone has been investigated in relation to SCS, further studies will have to confirm these data.

Beyond these segmental effects, cortical effects can be taken into account. ${ }^{23,24}$ In fact, it has been shown that HFSCS is more effective that tonic SCS in increasing intracortical facilitation and prolonging cortical silent period probed by TMS, thus suggesting a possible involvement of both GABAergic and glutamatergic networks at the intracortical level. ${ }^{20}$ As compared to these general effects, our data suggest a specific effect of HFSCS on the descending pain inhibitory pathways. In fact, we found an enhancement of PMI (ie, there was a reduction of MEP amplitude) when the stimulator was switched on. Such an enhancement further supports the involvement of A $\delta$ fiber-related inputs and potentially of the cortical areas involved in the sensory-discriminative and affective-emotional aspects of pain perception (subserved by the opercular cortex and the anterior cingulate gyrus, respectively), of which PMI is a functional marker. ${ }^{25}$ This descending modulation is consistent with the previously reported finding suggesting that SCS might not solely act by influencing pain associated with the neurobiological process at the spinal level but also by modulating excitability and neuroplasticity at the supraspinal level. ${ }^{19}$ Such cortical modulation could be NMDA-mediated, given that PMI modulation specifically depends on NMDA-related mechanisms. ${ }^{12}$ Moreover, these data further suggest that HFSCS may act on a wider network including both the lateral and medial pain pathways, whereas tonic SCS may principally modulate the lateral pain system. ${ }^{20}$

\section{Conclusion}

Even though our study could have benefited from long-term follow-up, future studies should be conducted to demonstrate the efficacy and safety of HFSCS (as the long-term benefits and outcomes are yet to be seen for HFSCS), supporting our findings and extending the treatment to other patients with these complex pain problems. More reports, case series, and randomized trials on these treatment modalities should be published to confirm their efficacy. Further, our case report 
offers a possible therapeutic option for patients with root avulsion, leading to paresthesia-free neuropathic pain reduction, which is proposed to depend on both large-diameter fiber block and medium-/small-diameter fiber recruitment, with a clear inhibition of WDR neurons.

\section{Author contributions}

DF contributed to conception and design of analysis, acquisition of data, and drafting of the article, and approved the final version to be published. FCerra contributed to conception and design of analysis, acquisition of data, and drafting of the article. GG, SM, NM, and AB involved in drafting of the article. FCorallo and AC participated in drafting of the article and acquisition of data. AN contributed to conception and design of analysis, interpretation of data, and drafting of the article. All authors contributed to data analysis, drafting or revising the article, gave final approval of the version to be published, and agree to be accountable for all aspects of the work.

\section{Disclosure}

The authors report no conflicts of interest in this work.

\section{References}

1. Chakravarthy K, Richter H, Christo PJ, Williams K, Guan Y. Spinal Cord Stimulation for Treating Chronic Pain: Reviewing Preclinical and Clinical Data on Paresthesia-Free High-Frequency Therapy. Neuromodulation. 2018;21(1):10-18.

2. Cruccu G, Garcia-Larrea L, Hansson P, et al. EAN guidelines on central neurostimulation therapy in chronic pain conditions. Eur J Neurol. 2016;23(10):1489-1499.

3. Russo M, Van Buyten JP. 10-kHz high-frequency SCS therapy: a clinical summary. Pain Med. 2015;16(5):934-942.

4. Urasaki E, Tsuda M, Nakane S, Toyoda K, Umeno T, Yamakawa Y. Spinal cord stimulation for intractable pain evaluated by a collision study using somatosensory evoked potentials: a preliminary report. Neuromodulation. 2014;17(8):746-752; discussion 752.

5. Huang YZ, Rothwell JC, Lu CS, et al. The effect of continuous theta burst stimulation over premotor cortex on circuits in primary motor cortex and spinal cord. Clin Neurophysiol. 2009;120(4):796-801.

6. Borsook D. Pain and motor system plasticity. Pain. 2007;132(1-2):8-9.

7. Inui K, Tran TD, Hoshiyama M, Kakigi R. Preferential stimulation of A $\delta$ fibers by intra-epidermal needle electrode in humans. Pain. 2002;96(3):247-252.

8. Mouraux A, Iannetti GD, Plaghki L. Low intensity intra-epidermal electrical stimulation can activate A $\delta$-nociceptors selectively. Pain. 2010;150(1):199-207.
9. Valeriani M, de Tommaso M, Restuccia D, et al. Reduced habituation to experimental pain in migraine patients: a $\mathrm{CO}(2)$ laser evoked potential study. Pain. 2003;105(1-2):57-64.

10. Mizuno Y, Tanaka R, Yanagisawa N. Reciprocal group I inhibition on triceps surae motoneurons in man. J Neurophysiol. 1971;34(6): $1010-1017$.

11. Crone C, Hultborn H, Mazières L, Morin C, Nielsen J, Pierrot-Deseilligny E. Sensitivity of monosynaptic test reflexes to facilitation and inhibition as a function of the test reflex size: a study in man and the cat. Exp Brain Res. 1990;81(1):35-45.

12. Suppa A, Biasiotta A, Belvisi D, et al. Heat-evoked experimental pain induces long-term potentiation-like plasticity in human primary motor cortex. Cereb Cortex. 2013;23(8):1942-1951.

13. Kapural L, Yu C, Doust MW, et al. Novel 10-kHz High-frequency Therapy (HF10 Therapy) Is Superior to Traditional Low-frequency Spinal Cord Stimulation for the Treatment of Chronic Back and Leg Pain: The SENZA-RCT Randomized Controlled Trial. Anesthesiology. 2015;123(4):851-860.

14. Linderoth B, Meyerson BA. Spinal cord stimulation: exploration of the physiological basis of a widely used therapy. Anesthesiology. 2010;113(6):1265-1267.

15. Buonocore M, Bonezzi C, Barolat G. Neurophysiological evidence of antidromic activation of large myelinated fibres in lower limbs during spinal cord stimulation. Spine (Phila Pa 1976). 2008;33(4):E90-E93.

16. Arle JE, Carlson KW, Mei L, Iftimia N, Shils JL. Mechanism of dorsal column stimulation to treat neuropathic but not nociceptive pain: analysis with a computational model. Neuromodulation. 2014;17(7):642-655; discussion 655.

17. Holsheimer J. Which neuronal elements are activated directly by spinal cord stimulation. Neuromodulation. 2002;5(1):25-31.

18. Urasaki E, Tsuda M, Nakane S, Toyoda K, Umeno T, Yamakawa Y. Spinal cord stimulation for intractable pain evaluated by a collision study using somatosensory evoked potentials: a preliminary report. Neuromodulation. 2014;17(8):746-752; discussion 752.

19. Buonocore M, Demartini L. Inhibition of Somatosensory Evoked Potentials During Different Modalities of Spinal Cord Stimulation: A Case Report. Neuromodulation. 2016;19(8):882-884.

20. Bocci T, De Carolis G, Paroli M, et al. Neurophysiological Comparison Among Tonic, High Frequency, and Burst Spinal Cord Stimulation: Novel Insights Into Spinal and Brain Mechanisms of Action. Neuromodulation. 2018;21(5):480-488.

21. Molnar G, Barolat G. Principles of cord activation during spinal cord stimulation. Neuromodulation. 2014;17(Suppl 1):12-21.

22. Arle JE, Mei L, Carlson KW, Shils JL. High-Frequency Stimulation of Dorsal Column Axons: Potential Underlying Mechanism of ParesthesiaFree Neuropathic Pain Relief. Neuromodulation. 2016;19(4):385-397.

23. De Ridder D, Vanneste S. Burst and tonic spinal cord stimulation: different and common brain mechanisms. Neuromodulation. 2016;19(1):47-59.

24. De Ridder D, Vanneste S, Plazier M, van der Loo E, Menovsky T. Burst spinal cord stimulation: toward paresthesia-free pain suppression. Neurosurgery. 2010;66(5):986-990.

25. Xie YF, Huo FQ, Tang JS. Cerebral cortex modulation of pain. Acta Pharmacol Sin. 2009;30(1):31-41.
Journal of Pain Research

\section{Publish your work in this journal}

The Journal of Pain Research is an international, peer reviewed, open access, online journal that welcomes laboratory and clinical findings in the fields of pain research and the prevention and management of pain. Original research, reviews, symposium reports, hypothesis formation and commentaries are all considered for publication.

\section{Dovepress}

The manuscript management system is completely online and includes a very quick and fair peer-review system, which is all easy to use. Visit http://www.dovepress.com/testimonials.php to read real quotes from published authors. 